\title{
Modal and path contribution models from in-operation data: Review and new approaches
}

\author{
H. Van der Auweraer*, P. Mas, B. Peeters, K. Janssens and A. Vecchio \\ LMS International NV, Interleuvenlaan 68, B-3001 Leuven, Belgium
}

Received 2007

Revised 2007

\begin{abstract}
In several cases, laboratory-based modelling approaches such as Experimental Modal Analysis and Transfer Path Analysis run into limitations with respect to technical and economical feasibility (accessibility of the structure, excitation feasibility, measurement time, accuracy, ...) as well as model representativity. Therefore, approaches such as in-operation modal analysis (OMA) have been developed and are reaching a similar degree of acceptability as standard EMA, with all limitations regarding accuracy and observability of the structural dynamics. In the same line of reasoning fits the question of system characterization and identification using (or based on) other operation data types such as transmissibility functions and coherence functions and data analysis procedures such as principal component analysis. Using these testing and analysis paradigms instead of the classical load-FRF-response one, key analysis techniques such as transfer path and contribution analysis can be reconsidered.
\end{abstract}

Keywords: Operational, modal, path, transmissibility, analysis

\section{Introduction}

Experimental models are a cornerstone in the development, refinement and troubleshooting process in a wide variety of industrial applications. The use of these models is based on a separation of the investigated noise or vibration problem into a source, a transfer and a receiver subsystem. The transfer system is then described by a model. For all possible loading conditions (sources), the impact at receiver level (the response) can be predicted.

The experimental procedure hereto consists of two parts: the assessment of the model in laboratory conditions and the collection of data required to derive operating loads. The latter is of course a measurement in operational conditions of the system under study. These model and load data then allow the investigation of the influence of particular system characteristics on the response (e.g. by evaluating the Frequency Response Functions and/or by extracting physically relevant model parameters of the transfer system, which is the basis for Experimental Modal Analysis - EMA). Alternatively, these data allow to evaluate the influence of particular loads on the response (which is the cornerstone of the Transfer Path Analysis -TPA-approach).

As a consequence, modelling approaches such as black-box Frequency Response Function Models, Experimental Modal Analysis and Transfer Path Analysis have become standard practices in the product development and engineering process.

However, in several cases, this laboratory-based approach runs into limitations with respect to technical and economical feasibility (accessibility of the structure, excitation feasibility, measurement time, accuracy, ...) as well as model representativity (linearization point ...). In other words, it may not be (physically) possible to extract

\footnotetext{
*Corresponding author. E-mail: herman.vanderauweraer@1msintl.com.
} 
the system characteristics through a separate set of laboratory measurements, or it may not be practically feasible because of cost and time constraints. It may even be technically not valid to do so (in case the actual system behaviour under operational conditions will differ from the one reproduced in the laboratory test).

Therefore, approaches such as in-operation modal analysis (OMA) have been developed. OMA allows extracting the dynamic system characteristics (including a modal parameter model) directly from the in-operation data without the need for an additional laboratory test. The OMA approach gets more and more introduced and is reaching a similar degree of acceptability as standard EMA [1]. In many applications, where it is feasible to do so, both techniques are even used in combination as their results in terms of representativity and accuracy complement each other and serve different analysis objectives.

A similar question can be raised for system characterization and identification using (or based on) other operation data types such as transmissibility functions and coherence functions and data analysis procedures such as principal component analysis. Using these testing and analysis paradigms instead of the classical load-FRF-response one, key analysis techniques such as transfer path and contribution analysis can be reconsidered.

The present paper addresses these issues for the practical case of transfer path analysis using only operational data. The corresponding theoretical description is reviewed, but attention is also focused on the practical implementation and usability issues such as sensors and test procedures but also the actual added value for solving a specific noise or vibration problem. Some practical case studies will illustrate the discussion.

\section{Operational spectral analysis}

Spectrum analysis is the basic approach to identify and understand the frequency components of an NVH problem. The interrelation between various signals, caused by the same source, can be visualized by mapping the spectra on a geometric model, provided that phase-consistent (or simultaneous) data are available. Such analysis is referred to as "Operational Deflection Shape" - ODS- analysis.

But in many applications, multiple sources are active at the same time. The key problem then consists of assessing the relevance (and contribution) of each source to the problem response [2,3]. In case the sources are uncorrelated, ordinary coherence techniques are used to quantify the degree of correlation between two signals and to separate the contributions. Provided that a reference signal, uncorrelated to the other sources, is available, Referenced Spectra describe the phase-consistent noise field of each source and a Coherent Power calculation identifies the partial contribution of each source to the total noise. In case multiple, partially correlated, sources are present the problem becomes more complex, requiring the use of Partial Coherence techniques (which are difficult to apply and interpret) and/or Principal Component Analysis techniques [3]. In the latter case, the original noise and vibration field is decomposed into contributions by independent contributions, coherent with "virtual" sources. Spectral as well as spatial (ODS) analysis can be applied on the "virtual components". While all approaches allow to identify global source contributions and spectral features, they however do not provide insight in individual transfer paths or system properties.

\section{Operational Modal Analysis}

\subsection{Operational Modal Analysis methodology}

Operational Modal Analysis requires processing operational response data directly into modal parameters [1].

Initially, time series modeling techniques have been applied in the field of OMA. However, the direct use of output time histories evolved to the use of output correlations as the latter methods are faster and more data can be analyzed due to the inherent data reduction step. It can be shown that a structure excited by white noise, yields output correlations that have similar mathematical expressions as Impulse Responses Functions (IRF). Therefore all classical (input-output) modal analysis methods that operate on IRFs can be transferred to the OMA case operating on autoand cross-correlation functions. An overview is given in Fig. 1. Within each class of methods (time history versus correlation based), there is also an evolution from Prediction Error Methods identifying ARMA models towards 


\begin{tabular}{|c|c|c|}
\hline \multicolumn{2}{|l|}{ Method } & Features \\
\hline \multirow[t]{2}{*}{$\begin{array}{l}\text { Output time } \\
\text { histories }\end{array}$} & $\begin{array}{l}\text { Stochastic time series } \\
\text { modelling; e.g. ARMA } \\
\text { models }\end{array}$ & $\begin{array}{l}\text { - Not practical for OMA } \\
\text { - No convincing cases in } \\
\text { literature }\end{array}$ \\
\hline & $\begin{array}{l}\text { Subspace identification } \\
\text { (BR, CVA, : } \\
\text { (bifferences very subtle) }\end{array}$ & $\begin{array}{l}\text { - Successful applications } \\
\text { - Limited number of samples / } \\
\text { channels, large computation } \\
\text { time }\end{array}$ \\
\hline \multirow{2}{*}{$\begin{array}{l}\text { Output } \\
\text { correlations }\end{array}$} & Polyreference LSCE & - Fast \\
\hline & Subspace identification & $\begin{array}{l}\text { - Data reduction step (time } \\
\text { series } \rightarrow \text { correlations) } \\
\text { - Relatively fast }\end{array}$ \\
\hline
\end{tabular}

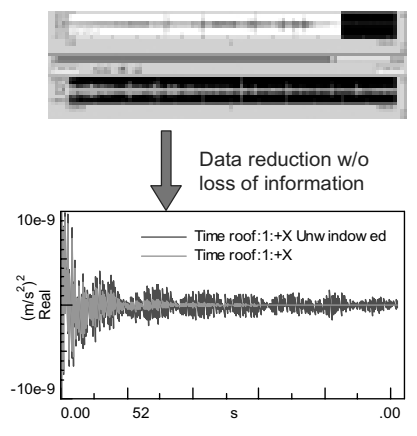

Fig. 1. Evolution in time-domain OMA methods. Parameter estimation (left), data reduction (right).

\begin{tabular}{|l|l|l|}
\hline Method & Features \\
\hline \multirow{5}{*}{$\begin{array}{l}\text { Non- } \\
\text { parametric }\end{array}$} & Peak picking / ODS & $\begin{array}{l}\text { - Subjective } \\
\text { - No damping }\end{array}$ \\
\cline { 2 - 3 } & $\begin{array}{l}\text { SVD-based } \\
\text { - PCA, CMIF, FDD } \\
\text { - Can be complimented } \\
\text { by simple SDOF curve- } \\
\text { fitters }\end{array}$ & $\begin{array}{l}\text { - Still picking peaks } \\
\text { (subjective / difficult) } \\
\text { - Better than PP for closely } \\
\text { spaced modes } \\
\text { - SDOF extension highly } \\
\text { interactive and tedious }\end{array}$ \\
\hline Parametric & PolyMAX & $\begin{array}{l}\text { - Clear stabilization } \\
\text { - Highly automatic }\end{array}$ \\
\cline { 2 - 3 } & $\begin{array}{l}\text { (Polyreference) } \\
\text { Maximum Likelihood }\end{array}$ & $\begin{array}{l}\text { - Stochastic (incl. noise } \\
\text { information) } \\
\text { - Iterative }\end{array}$ \\
\hline
\end{tabular}

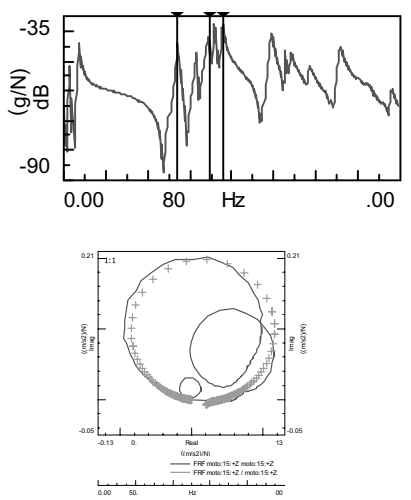

Fig. 2. Evolution in frequency-domain OMA methods. Parameter estimation methods (left); Illustration of peak-picking and simple SDOF curve-fit (circle-fit) (right).

Subspace identification and from Polyreference LSCE again to Subspace identification [4]. Subspace identification comes in two classes: either time series based or correlation-based. Also within each class, many variants exist: Balanced Realization (BR), Canonical Variate Analysis (CVA), . . but their differences are very subtle and do not seem to be relevant in modal analysis applications.

With frequency-domain methods, the main evolution is from non-parametric to parametric methods (Fig. 2). Non-parametric involves signal processing techniques (FFT, SVD, ...) to enhance certain signal features (spectral peaks); the user must select modes based on these peaks. This is a subjective process, inspecting many spectra to find all modes and with coupled modes or noisy data, the peaks may not be clear. SVD-methods, enhancing modal appearance, may be complemented with SDOF estimators, which is a time-consuming process.

Parametric analysis means that a model is fitted to the data. Parametric methods have the great advantage that stabilization diagrams, fitting models of different order, can be used to objectively find the modes. An example of a parametric method that received considerable attention recently is the PolyMAX method [5]. Its main benefit is that it yields extremely clear stabilization diagrams and thus that it has potential to be used as an autonomous modal parameter selection method. More advanced iterative estimators like Maximum Likelihood have the advantage that uncertainty on the data is taken into account in the identification process and that confidence bounds on the modal parameter estimates are available. PolyMAX can be viewed as an already excellent starting value generator for these methods. 


\subsection{Application of operational modal analysis}

The need to perform operational or output-only modal analysis probably emerged first in civil engineering, where it is very difficult and expensive to excite constructions such as bridges and buildings with a hammer or shaker and to obtain artificially induced vibration levels that exceed the natural vibrations due to traffic or wind. Nevertheless, also the benefits of OMA in mechanical engineering became clear: for instance to study the road behaviour of cars, and to analyze data from aircraft excited by in-flight turbulences.

A typical aerospace application is the exploitation of flight data using Operational Modal Analysis. The reason for using techniques that do not require input information is that it is difficult to measure the forces from the artificial excitation devices and that these techniques also allow the use of natural turbulences as input. Flight tests take place at the end of the development cycle of a new aircraft and allow the validation of the analytical models under various real flight conditions. More importantly, they allow assessing the aero-elastic interaction, as a function of airspeed and altitude, between the structure and the aerodynamic forces as they may lead to a sudden unstable behaviour known as flutter. Under no condition should an aircraft structure enter into flutter and it has to be certified that it has sufficient flutter margin when flying at the different points of the flight envelope where it is designed for. To determine the flutter margin, the trends of eigenfrequencies and damping ratios of the critical modes as a function of airspeed are carefully studied. This explains the need to perform a modal analysis during the flight. More information can be found in [6-8]. OMA is also applied to in-flight helicopter testing [9], where one of the challenges is the separation between rotor harmonics and structural modes.

Also in automotive engineering, OMA has been applied. In troubleshooting, competitive benchmarking, or target setting projects, it is common to acquire a lot of vibration data while the car is driven on the road or tested on a roller bench or a 4-poster [10]. A major concern in the development of new cars is the acoustic comfort for driver and passengers. Interior vehicle sound plays an important role in brand identification and product differentiation. There are various sound characteristics that contribute to a typical brand sound. Resonances, amplitude modulations, order non-linearities, booming and masking effects are typical examples of important acoustic features. Recently, a new procedure was developed to automatically identify resonances from (operational) engine run-up data [11]. Some other applications of OMA are the validation of assembly models of wind turbines [12] and the study of the behaviour of industrial machine vibrations $[13,14]$.

\section{Operational path analysis}

The best known source-system-transceiver approach to analyze NVH problems is the Transfer Path Analysis method [15]. While yielding very useful data, accurate measurement procedures require extensive laboratory tests in addition to in-operation tests. Recently, extended and alternative approaches were proposed to reduce measurement time while keeping most of the analysis advantages of the TPA method [16]. Some approaches complement the in-operation tests with limited extra FRF tests; some solely use in-operation measured data.

\subsection{Basic TPA formulation}

Transfer Path Analysis (TPA), Noise Path Analysis (NPA), Path or Source Contribution Analysis ... refer all to one of the most important NVH analysis approaches of the last decennia, expressing an investigated noise or vibration response as a sum of contributions due to individual paths and/or sources. The classical approach to this problem was to use coherence analysis to identify the various contributions [2,3]. In the late 80 -ies, an alternative formulation was developed making use of a source-system-receiver model, expressing the response as a sum of contributions, each being a system response resulting from an individual load (structural or acoustic), acting at a localized interface [15]. In the case of a pressure response, the key formula becomes:

$$
p(\omega)=\sum \frac{p}{F_{i}}(\omega) \cdot F_{i}(\omega)+\sum \frac{p}{Q_{i}}(\omega) \cdot Q_{i}(\omega)
$$

with $F_{i}$ and $Q_{i}$ resp. the structural and the acoustic load and $\mathrm{p} / \mathrm{F}$ and $\mathrm{p} / \mathrm{Q}$ the noise transfer functions. Similar equations hold for vibration responses (seat or steering wheel vibrations ....). This approach essentially corresponds 

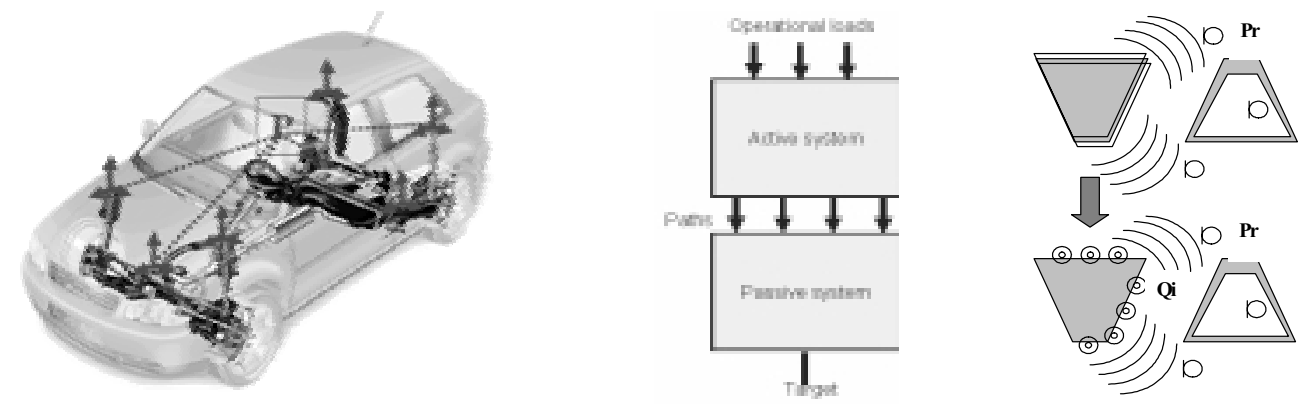

Fig. 3. TPA model: Vehicle suspension source-system separation (left), Active/passive system decomposition (middle), Discretized acoustic source model (right).

to "cutting" the global system at the interface into an "active" part generating the interface load and a "passive" part reacting to the interface load (Fig. 3). For structural loads, this "cut" typically corresponds to physical connection points (bushings, subsystem connections ...), for acoustic loads from vibrating surfaces or pulsations from nozzles or apertures, a discretization by omnidirectional volume acceleration point sources is applied [15]. Concise visualizations in the form of spectra, vector plots, geometric projections (in particular for airborne panel contribution analysis) etc. allow to quickly assess critical paths and frequency regions.

The two steps of the test procedure required to build the "TPA model" are: Eq. (1) to identify the operational loads during in-operation tests such as road tests, chassis dyno tests, and Eq. (2) to estimate the noise transfer functions, typically under laboratory conditions. The procedure is similar for both the structural and the acoustical loading cases, but the practical implementation is of course governed by the nature of the signals.

Very important to understand the complexity of the procedure is that FRF tests are not only needed to estimate the system transfer functions, but, and in even larger amounts, for the load identification step. Ideally, this load identification could be done using dedicated sensors, but this is not feasible in most measuring situations. Indirect procedures have hence been developed using either the differential operational responses across the connection mount (and the 3D mount stiffness characteristics), or a matrix inversion procedure using the responses at the passive system side and an impedance (force/acceleration) FRF matrix (sometimes referred to as "apparent mass") between all connection points and closeby responses. Both procedures have been discussed extensively in literature [17]. During the 90-ies, several advances have been proposed. Reciprocal measurements for the Noise Transfer Functions have significantly reduced measurement effort and improved accuracy [18]. Special sound sources have been developed hereto. The extension with Singular-Value-Analysis based Principal Component Analysis (PCA) [3], allowed the study of multivariate problems such as road noise, decomposing the coupled $\mathrm{N} \& \mathrm{~V}$ field into independent components to be modeled individually [19]. The result is that TPA analysis has become a widely used approach, in most cases used in combination with complementary tools such as coherence (and PCA) analysis, Operational Deflection Shape (ODS) analysis, modal analysis, and OMA.

Still, a main problem of TPA, and in particular the indirect technique, is the large measurement time for the FRF matrices. Therefore, reduced approaches measuring only part of the -in theory full- FRF matrix, are sometimes applied, neglecting the cross-coupling effects between the interface locations. This may involve a simplification to a block-diagonal matrix, only measuring cross-FRFs on the same subsystem, or only the cross-FRFs at each mount ( $3 * 3$ blocks) or even a full diagonal matrix, only considering the local impedance. However, the thereby introduced errors on the loads may be major, in particular in the full-diagonal approach. As a principle, the weaker various subsystems are connected, the lower the impact of their cross-coupling effects.

\subsection{Improved speed with fast and multilevel TPA}

In many NVH applications, a first and key question is to identify which of the different subsystems are the main contributors to the noise rather than identifying in detail each of the paths and loads. Examples are to assess air-vs-structure borne noise, front-vs-rear suspension, engine mounts versus other PT mounts ... For this purpose, a fast testing procedure [16] was developed complementing the traditional troubleshooting in-operation measurements 

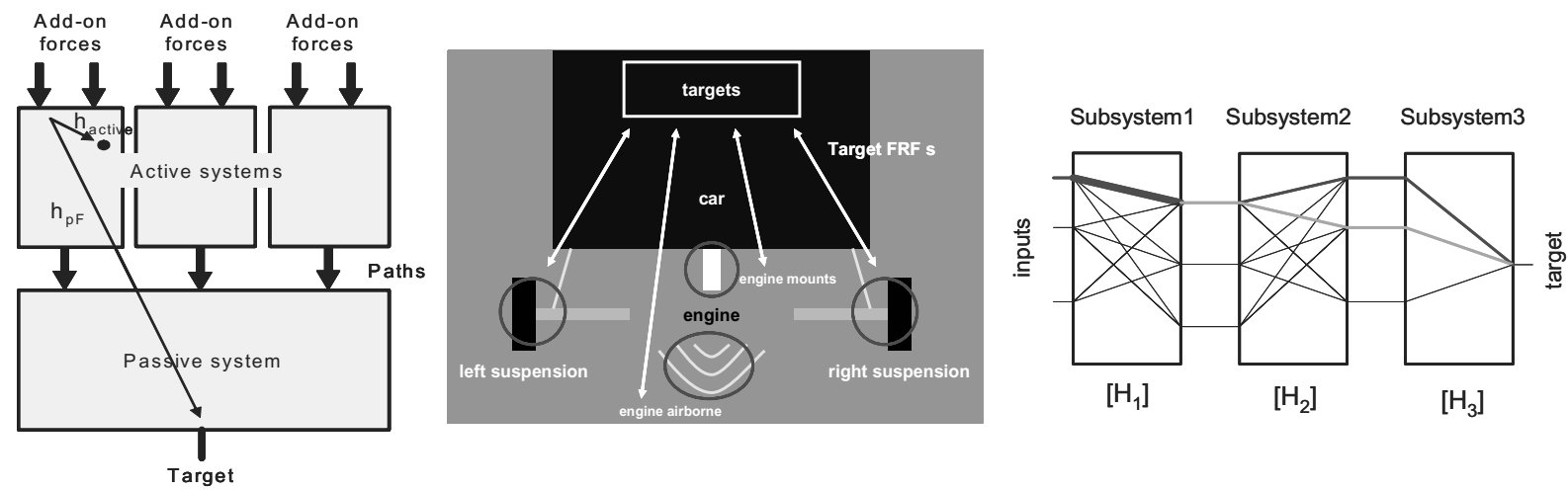

Fig. 4. Advanced TPA methods: Fast TPA (left/middle), Multilevel TPA (right).

(at subsystem locations and at target locations) by a limited number of additional FRF tests. These FRF are defined for the same response locations (and hence using the same instrumentation) as in the operational tests and for a limited set of arbitrary excitation points on the concerned subsystems, leading to a matrix $\mathrm{H}_{\text {active }}$. Combining this matrix with the operational responses $\{a\}$ allows to identify a set of "virtual" operational add-on loads which can then be used in a forward contribution analysis applying a noise transfer matrix $\mathrm{H}_{p a}$ (Fig. 4):

$$
\left\{F_{\text {add-on }}\right\}=\left[H_{\text {active }}\right]^{-1}\left\{a_{\text {active }}\right\} \Rightarrow\left\{p_{\text {contrib }}\right\}=\left\{H_{p F}\right\}\left\{F_{\text {add-on }}\right\}
$$

The summed response over all loads on a subsystem then represents the subsystem contribution to the noise.

"Fast TPA" is considered to be a very valid alternative for these applications where a fast assessment of the main contributing sources or subsystems is needed. It reuses existing instrumentation, involves limited extra testing, does not require building out the active systems and is compatible with the way of working of NVH analysts performing ODS and TPA studies. While it does not allow to derive the actual loads at the interfaces, the virtual add-on loads can be transferred to a CAE model and used for response calculations.

The Fast TPA concepts can be extended to study the contribution of components to a target response in a "chain" of linked subsystems in the noise path (Multilevel TPA [16], Fig. 4, right). The basic measurement is again a series of FRF measurements for a number of add-on forces close to the sources, whose component-level responses act as loads on the next subsystem level. The result is a series of force transmissibility matrices:

$$
\{p\}=\left[H_{1}\right]\left[H_{2}\right]\left[H_{3}\right] \cdots\{F\}
$$

$\left[\mathrm{H}_{1}\right]$ is a "classical" FRF (load-to-noise) matrix; $\left[\mathrm{H}_{2}\right] \ldots$ are component transmissibility matrices, for example force transmissibilities. The actual measurement effort remains limited compared to a full TPA. Reciprocal excitation through improved inertia shakers significantly simplifies the FRF test job.

\subsection{Operational path analysis}

One major constraint of all discussed TPA approaches is that, next to the in-operation response measurements (road tests, chassis dyno tests, road simulators ...), an extensive additional measurement campaign is needed to obtain the FRFs for the inverse force identification as well as for the propagation FRFs (NTF). Consequently, significant research has been conducted on methods which maximally exploit the in-operation measured data for deriving TPA-like results without requiring a lab test. This is very similar to the modal analysis research that led to Operational Modal Analysis. One can also note a renewed interest in coherence-related techniques to isolate source contributions. Applied to the Path Analysis problem, the basic operational model formulation is this of a response expressed in terms of other responses (but closer to the sources). For example, for an acceleration response (steering wheel), this output acceleration can be expressed as a sum of acceleration contributions [20]:

$$
\ddot{X}_{r}=\sum_{k} \ddot{X}_{k} H_{k r}
$$



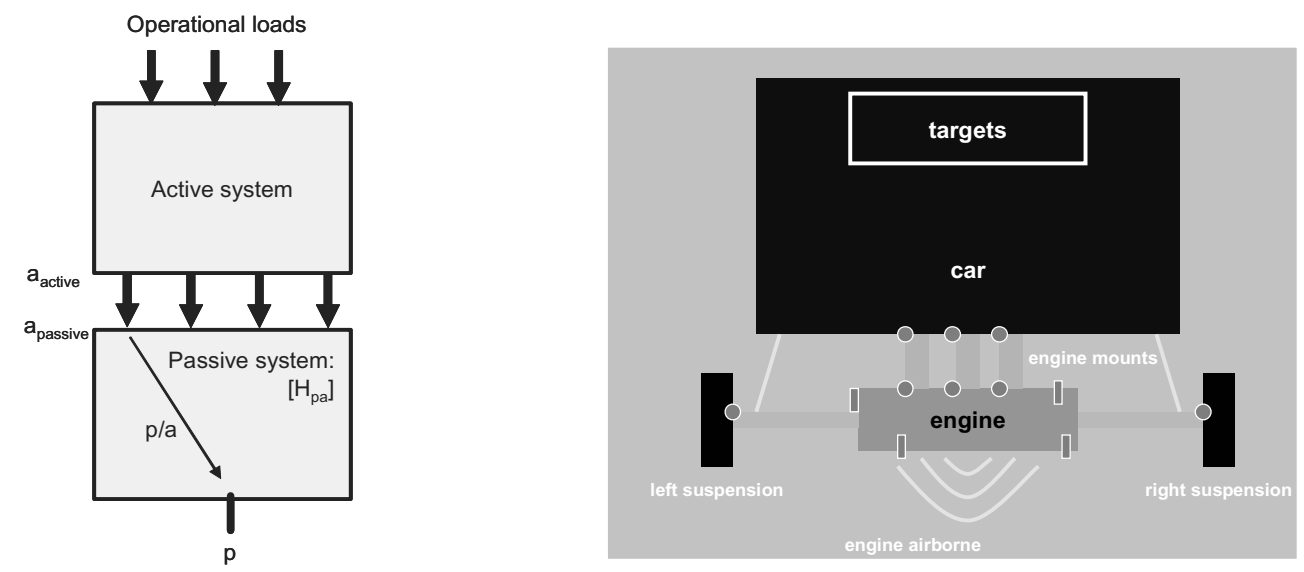

Fig. 5. Operational Path Analysis (OPA) scheme (left). Engine OPA scheme (right).

with $\ddot{X}_{k}$ contact acceleration at interface point $k$ and $H_{k r}$ transmissibility between input $k$ and output $r$.

Similar relations can be written for an acoustic response and/or acoustic references (near sources) [20], leading to a response model referred to as "Operational Path Analysis (OPA)", Fig. 5.

The methodology very closely resembles this of coherent output power calculation [21]. The key step in the method is the calculation of the MIMO transmissibility matrix H which is defined as the relation between an "input" and an "output" provided all other inputs are at rest. The procedure to obtain such transmissibility matrix from response measurements was extensively studied by Ewins and Maia [22-24]. The basic principle is very similar to this of any other FRF matrix, applying for example an $\mathrm{H}_{1}$ approach this gives:

$$
p=\sum_{\text {paths }} \frac{p}{a} \cdot a \Rightarrow\{p\}=\{a\} \cdot\{H\} \Rightarrow\langle a * p\rangle=\{H\} \cdot\langle a * a\rangle \Rightarrow\{H\}=\langle a * a\rangle^{-1} \cdot\langle a * p\rangle
$$

$\{\mathrm{p}\}$ is the target response vector; $\{\mathrm{a}\}$ is the vector with reference signals measured at critical interfaces, (both accelerations and pressures). These references should preferably be located near actual sources to provide maximal uncorrelation (e.g. engine-side mount locations, engine surface vibrations, exhaust/intake nozzles ...) in the subsequent contribution calculation (the transmissibility calculation inherently decorrelates the transfer functions, but not the sources itself).

The basic condition for performing this operation is the invertability of the reference autopower matrix [a*a], requiring that in the build-up (averaging) of the power spectra, at least a number of different conditions equal to the number of references are present at each frequency. This can in principle be obtained by combining tests under different conditions. E.g. during an engine run-up, each frequency will, with increasing RPM, be excited by different orders. Each order causes a different phase relation between the engine mount responses. Averaging over the RPM range hence decorrelates the autopower matrix. Tests with varying torques, or in different driving conditions can also help uncorrelate the transmissibility. Still, strong modal responses of the sources (engine rigid body ...) may still cause rank deficiency. Pseudo inverse procedures using optimization, $\mathrm{QR}$ decomposition or singular value (principal component) decomposition can then be applied [3,19].

The fact that both acoustic and structural responses can be used as references (e.g. for assessing structure versus air borne contributions) requires a proper balancing of the response quantities before performing the matrix inversion. This is similar to the well-known TPA and strain or vibro-acoustic modal analysis problem.

A related implementation of such approach, acting directly on the spectra, is described in [25]. In [26], a similar approach is interpreted in terms of energetic contributions.

Although the methodology is very appealing due to the absence of laboratory measurements, one has to be careful to derive "causal" interpretations as from a true Transfer Path Analysis. Being related to an elaborated coherence analysis providing insights into the subsystem and path responses dominating the target responses, the method rather provides "co-existential" information and is therefore referred to as "Operational Path Analysis". 

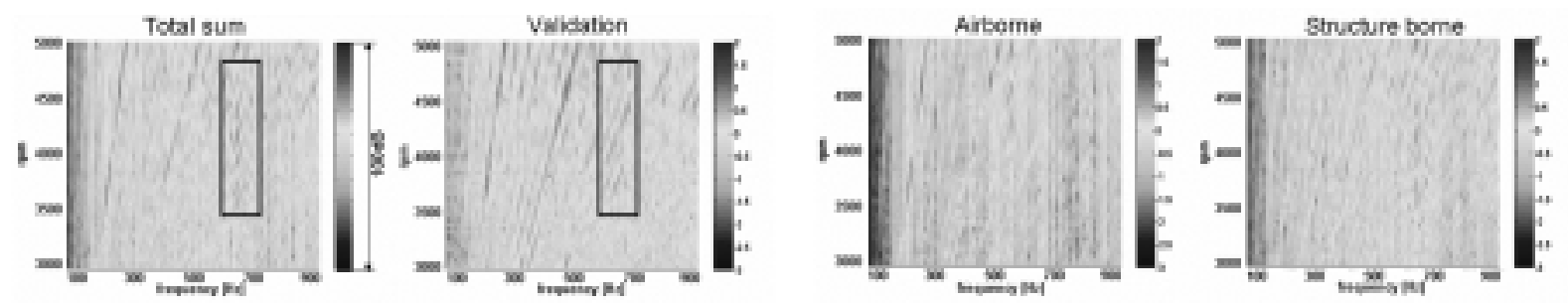

Fig. 6. Engine noise OPA waterfall (engine run-up) results: Total sum of the different paths (left), measured microphone (middle-left), airborne contribution (middle-right), structure borne contribution (right).

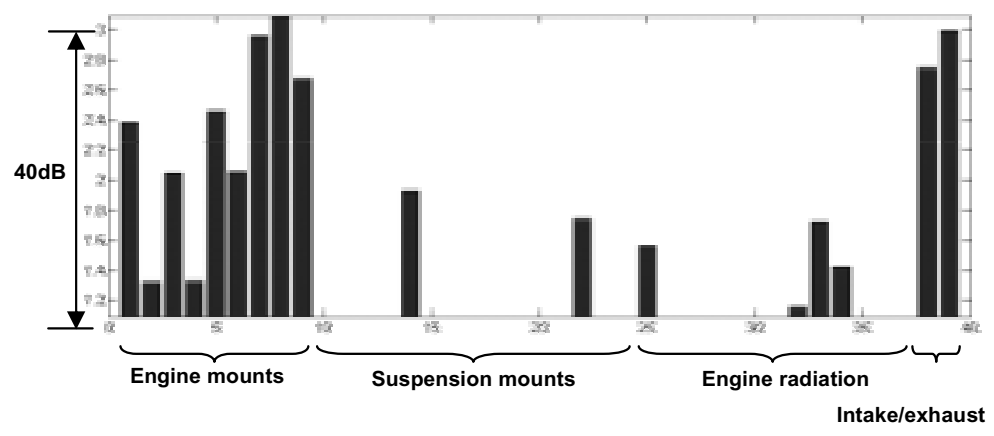

Fig. 7. OPA-based engine noise contribution analysis.

When assessing the approach by comparing summed synthesized responses with measured ones, care has also to be taken. As the same data are used in the backward (transmissibility) and forward (contribution) calculations, good similarity only allows qualifying noise influences and transmissibility matrix conditioning. For the same reason, the method is very sensitive to unmeasured reference contributions. These will be distributed over the measured ones, which in case of major "missed" sources, may drown the original values of weaker contributions. Great care has hence to be taken in interpreting the results.

Figure 5 (right) shows the schematic representation for an engine noise source separation study. Operational run-up measurements included target responses as well as accelerations near the active and passive engine mounts, at the wheel knuckles and driveshafts and at the main airborne sources. Figure 6 shows the key results.

The contribution analysis can be done for the orders, but easier to interpret is to calculate overall path contributions for bands of the complete waterfall. Based on a selection in the waterfall spectrum (order or band) a contribution plot of the different paths can then be constructed. Figure 7 shows the contribution for a band selected in the waterfall. It shows that the engine mounts are the main contributors to the sound and that also intake and exhaust contribute to a certain extent.

\section{Conclusion}

In the last 15 years, FRF modeling, Experimental Modal Analysis and Transfer Path Analysis have known a breakthrough as very relevant NVH troubleshooting tools. The main bottleneck of the "full" FRF, EMA or TPA method is the required test time to build the full data model by dedicated laboratory tests. Several new approaches that try to circumvent or reduce this limitation are being developed. Insight in the actual formulations, accuracy constraints and application limitations is however a prerequisite in order to allow correct interpretation and validation of the thus obtained results. Operating Deflection Shape Analysis complements FRF analysis, OMA methods are a valid complement to EMA and, recently, Fast TPA and Operational Path Analysis techniques have been proposed that may bring an important contribution to TPA test simplification. 


\section{Acknowledgements}

The discussed results have been obtained as part of several international research projects funded by the European Commission (DIANA, PIANO, SALOME) and IWT Vlaanderen (Virtual Car Sound). Current research is funded by the EC Marie Curie project "Smart Structures" and IWT Project "Acoustic Brush".

\section{References}

[1] B. Peeters, W. Leurs, H. Van der Auweraer and F. Deblauwe, 10 years of industrial Operational Modal Analysis: evolution in technology and applications, Proc. IOMAC 2006, 4-5 May 2006, Aalborg (DK).

[2] J.S. Bendat and A.G. Piersol, Engineering Applications of Correlation and Spectral Analysis, Wiley, New York, 1980.

[3] D. Otte, Development and Evaluation of Singular Value Analysis Methodologies for Studying Multivariate Noise and Vibration Problems, Ph. D dissertation, K.U.Leuven (B), 1994.

[4] L. Hermans and H. Van der Auweraer, Modal Testing and Analysis of Structures under Operational Conditions: Industrial Applications, Mechanical Systems and Signal Processing 13(2) (1999), 193-216.

[5] B. Peeters, H. Van der Auweraer, P. Guillaume and J. Leuridan, The PolyMAX Frequency-Domain Method: A New Standard for Modal Parameter Estimation? Shock and Vibration, Special Issue dedicated to Professor Bruno Piombo 11 (2004), 395-409.

[6] M.W. Kehoe, A Historical Overview of Flight Flutter Testing, NASA TM-4720, www.dfrc.nasa.gov/DTRS, 1995.

[7] B. Peeters, A. Vecchio and H. Van der Auweraer, PolyMAX Modal Parameter Estimation from Operational Data, Proceedings of the ISMA 2004, Leuven, Belgium, 20-22 September 2004.

[8] C.R. Pickrel and P.J. White, Flight Flutter Testing of Transport Aircraft: In-Flight Modal Analysis, Proceedings of IMAC 21, Kissimmee (FL), USA, 2003.

[9] C. Cavagnino, Operational Modal Analysis of a Lightweight Helicopter Tail, LMS Conference Europe, Minchen, Germany, 22-23 March 2006.

[10] R. Jöst, Extension of the Development Potential of Car Body Structures by using High Power Electrodynamic Shakers, LMS Conference Europe, München, Germany, 22-23 March 2006.

[11] K. Janssens, Z. Kollar, B. Peeters, S. Pauwels and H. Van der Auweraer, Order-Based Resonance Identification using Operational PolyMAX, Proc. of IMAC 24, Saint Louis (MO), USA, 30 January - 2 February 2006.

[12] L. Bonnet, Wind Turbine Acoustic Modeling, LMS Conference Europe, Nürburg, Germany, 2-3 March 2005

[13] A. Harms, Increasing the Dynamic Stiffness of Machine Tools by Means of Modal Analysis, LMS Conference Europe, Minchen, Germany, 22-23 March 2006.

[14] A. Vecchio and M. Scionti, A Modal Based Approach to Assess Dynamic Behaviour of Structural Alternatives in Machinery, Proceedings of ISMA 2002, Leuven, Belgium, September 2002.

[15] D. De Vis, W. Hendricx and P. van der Linden, Development and Integration of an Advanced Unified Approach to Structure Bome Noise Analysis, 2nd Int. Conference on Vehicle Comfort, ATA, 1992.

[16] H. Van der Auweraer, P. Mas, S. Dom, A. Vecchio, K. Janssens and P. Van de Ponseele, Transfer Path Analysis in the Critical Path of Vehicle Refinement: The Role of Fast, Hybrid and Operational Path Analysis, Submitted to SAE Noise and Vibration Conf. May 2007.

[17] P. van der Linden and H. Floetke, Comparing Inverse Force Identification and the Mount Stiffness Force Identification Methods for Noise Contribution Analysis, Proc. 2004 ISMA, Sept. 2004, Leuven (B).

[18] P. van der Linden and J. Fun, Using Mechanical-Acoustic Reciprocity for Diagnosis of Structure Borne Sound in Vehicles, SAE 93130.

[19] L. Gielen and R. Deges, The Identification, Quantification and Reduction of Structural-Borne Road Noise in a Midsize Passenger Car, SAE Int. Congress and Exposition, Feb. 26-29, 1996, Detroit, (MI) USA.

[20] P. van der Linden and K. Wyckaert, Modular Vehicle Noise and Vibration Development, Proc. SAE Noise and Vibration Conference, Traverse City (MI), May 1999.

[21] W. Halvorsen, Noise Source Identification using Coherent Output Power Spectra, Sound and Vibration, Aug. 1975, 17-24.

[22] D. Ewins and W. Liu, Transmissibility Properties of MDOF Systems, Proc. 16th IMAC, Santa Barbara (CA), pp. 847-854, California, 1998.

[23] N.M.M. Maia, J.M.M. Silva and A.M.R. Ribeiro, The Transmissibility Concept in Multi-degree-of-freedom Systems, Mechanical Systems \& Signal Processing 15(1) (January 2001), 129-137.

[24] A.M.R. Ribeiro, J.M.M. Silva, N.M.M., Maia and M. Fontul, Transmissibility Matrix in Harmonic and Random Processes, $J$ of Shock and Vibration 11(5-6) (2004), 563-571.

[25] K. Noumura and J. Yoshida, Method of Transfer Path Analysis for Vehicle Interior Sound with no Excitation Experiment, Proc. FISITA 2006, F2006D183, Yokohama (JP), October 2006.

[26] A. Vecchio and H. Van der Auweraer, Vibroacoustic Characterization of a Hybrid Car, Proc. 11th Int. Congress on Measuring and Testing Techniques in Vehicle Development, Apr. 3-4, 2003, Würzburg (D). 

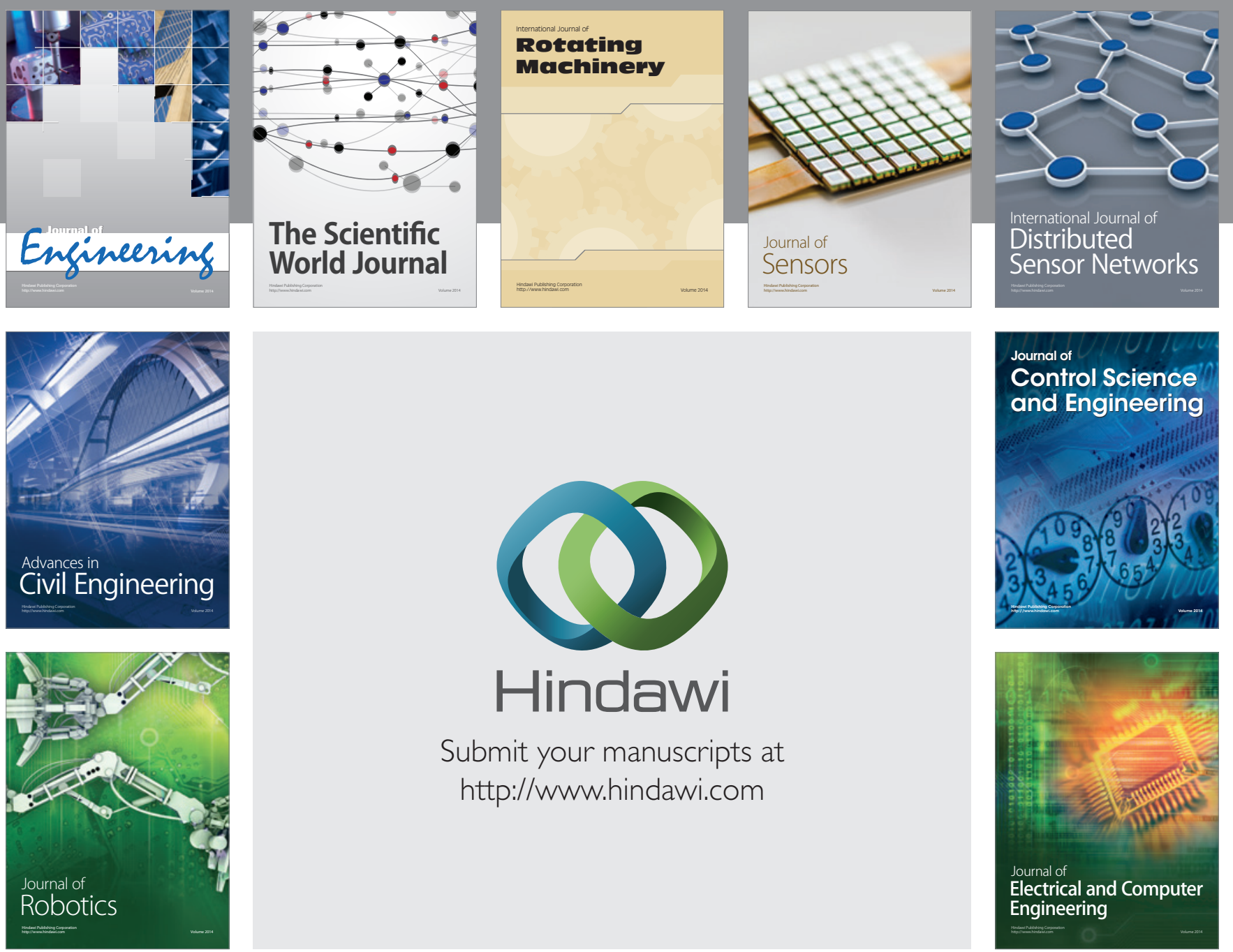

Submit your manuscripts at

http://www.hindawi.com
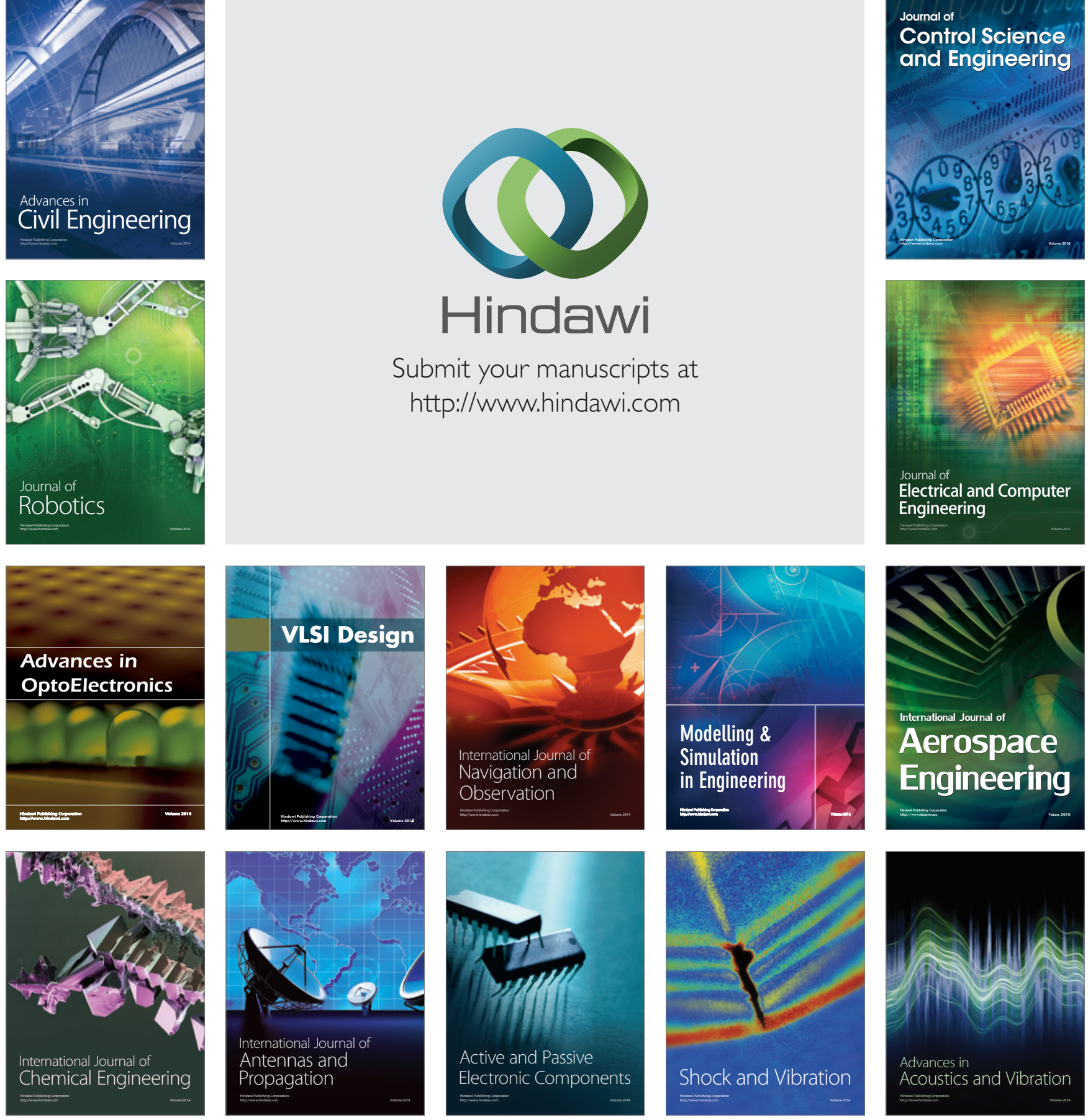